\title{
Design and Implementation of Therapist Online Counseling
}

\author{
Aaron O. Thomas, Geoff Lee and Brian Ess \\ University of Florida
}

\begin{abstract}
The following paper describes the rationale for creating an online therapy program of cognitive behavioral therapy, or CBT, for students at the University of Florida who are struggling with stress and anxiety. In addition, the paper discusses the unique challenges of the instructional design process of creating psychoeducational materials through cognitive task analysis and heuristics. Preliminary evidence suggests that higher education institutions may wish to implement similar programs in order to increase efficiency and serve both on-campus and distance students.
\end{abstract}

In recent years, college and university counseling centers nationwide have reported a trend toward greater student distress and a corresponding increase in counseling services utilization (American College Health Association, 2014; AUCCCD, 2013). Commonly used methods of attempting to meet increased demand for services (i.e., increase therapist caseloads, reduce session frequency, waitlists) are often associated with less than optimal outcomes for students accessing them, while also leaving many more students without the treatment they need (Gallagher, 2011). Given the trend toward increased use of technology in the academic and personal lives of students, we decided to develop a treatment solution that leverages computer-based learning environments, text messaging, and synchronous video conferencing to support clients who may need clinical support services but who live at a distance or cannot find the time to meet at a therapist's office. Because of privacy and confidentiality concerns, we did not believe that social media was an appropriate tool to use in the context of telemental health. We labeled this approach Therapist-Assisted Online (TAO), based on international treatment models, which combine online psychoeducational treatment materials with brief therapist contact. In contrast to online self-help alone, therapist-assisted treatments result in increased client treatment, engagement, compliance, and completion rates (Andersson \& Cuijpers, 2009; Berger et al., 2011; Spek et al., 2007). Despite the limited nature of this contact, clients report high levels of treatment satisfaction and therapeutic alliances comparable to traditional counseling (Andrews, Cuijpers, Craske, McEvoy, \& Titov, 2010). Another aspect that makes 
TAO effective for our students is that it utilizes cognitive behavioral therapy (CBT), a well-researched and highly effective treatment approach (Butler, Chapman, Forman, \& Beck, 2006). We chose the cognitive behavioral approach because it is easily adapted for online use with its structured, directive, and psychoeducational format, while encouraging active participation in the treatment process (Mureşan, Montgomery, \& David, 2012).

The following paper will discuss the theoretical frameworks and processes that guided the design process in order to provide a potential solution for wellness services to other educational institutions wishing to meet the increasing needs of both on-campus and distance students. The ecosystem of tools used in the implementation of TAO was complex, including a modified learning management system, external texting service, videoconferencing tool, and an iOS monitoring log. The design and implementation of each tool required extensive research into the affordances and limitations of the technologies along with a keen awareness of the Health Insurance Portability and Accountability Act (HIPAA).

\section{Online Therapy and College Counseling}

In the spring of 2011, the University of Florida's Counseling and Wellness Center (CWC) and College of Education's distance education learning unit collaborated on the design, development, and implementation of web-based psychoeducational materials that were to be delivered through a learning management system (LMS) that had been modified to support SCORM 2004 and WebRTC videoconferencing. The rationale for launching the project stemmed from a need at the CWC to increase clinical capacity by leveraging technology as a way to offload common psychoeducational lessons traditionally delivered during the client face-to-face sessions. Because demand for counseling and wellness services in the postsecondary environment continues to grow without a proportionate growth in clinical staff and because postsecondary school students appear to be receptive to this type of treatment, programs like TAO offer a means to meet increasing demand (Travers \& Benton, 2014). The CWC clinical team hypothesized that approximately 30 minutes of web-based instructional content and 15 minutes of video conferencing each week would be a therapeutic equivalent to a typical hour-long session where clients come to a traditional office for face-to-face therapy. If a TAO therapist could serve two to three clients per hour, the TAO approach promised to become a tool to address institutional needs to expand clinical capacity to the student body.

\section{Design Model}

Effective instruction is designed and intentional in terms of its goals, activities, and implementation (Morrison, Ross, Kemp, \& Kalman, 2010). In a traditional model commonly used in the military and large corporations, a linear process of needs analysis, design, development, implementation, and evaluation (ADDIE) is employed to create a final instructional product (Dick, Carey, \& Carey, 2005). In the case of TAO, however, we employed a successive approximation model (SAM) rather than the traditional ADDIE model, which is far more rigid and time-consuming (Allen \& Sites, 2012). SAM allowed us to shorten our development cycles so as to provide helpful materials to clients faster and receive feedback sooner from both clients and therapists. In the first year of the TAO pilot, four major updates were created to better meet the needs of both clients and therapists as feedback from both therapists and student clients was gathered.

The dynamic and flexible approach to this process was a great asset. In the case of TAO, the instructional designer surveyed the clients through formal surveys to evaluate user satisfaction, technical bugs, and usability information. These surveys were housed within the secure, HIPAA-compliant LMS that we had developed for mental health care applications. In addition, the clinical team shared with the production team qualitative feedback that clients had provided as they worked their way through TAO. Client satisfaction surveys indicated greater satisfaction with each iteration of the TAO materials that slowly evolved from traditional PowerPoint slides to interactive multimedia presentations that were closely designed in accordance with Mayer's (2009) cognitive theory of multimedia learning (CTML). 
CTML was a useful guide in evaluating the degree to which the interactive multimedia lessons would impact cognitive load and the meaning-making process.

\section{Generic Therapeutic Scaffolds}

Educational research has shown that learning is an active process where learners are meaning builders rather than receptacles for units of information (Jonassen, 1999; Mayer, 2009).

Because of our constructivist and cognitivist orientations, the TAO team decided to build generic instructional and therapeutic scaffolds that have been shown to improve a learner's objective performance and self-regulatory skills (Belland, 2014). These scaffolds were designed to better align the clients' understanding of content material with the instructional goals, to adapt to clients' responses, and to encourage the transfer of responsibility to the clients (Puntambekar \& Hubscher, 2005).

For example, the goal-setting lesson was broken down into a series of steps in which clients describe their current goal setting behaviors, evaluate the effectiveness of these behaviors using rubrics designed by the clinical team, and then construct specific goals for their therapy in a step-by-step process. At the end of this process, clients were then required to review their responses and evaluate the reasonableness of their goals. This type of self-reflective activity is common throughout TAO, where clients do not merely receive mini-lectures on a topic but are also required to work through the topic by answering questions, engaging in role-playing scenarios, and evaluating their responses and behavior within the TAO system. In addition, the monitoring logs or self-reflective journals were designed to allow clients to evaluate their progress and identify patterns that could be addressed in the videoconference meeting with their therapist.

The therapist also had access to these online journals and a record of client progress through each activity. Client monitoring of program adherence by the therapist was a great asset that we believe may have resulted in greater adherence to treatment guidelines and practice. As compared to many traditional counseling environments where there is no ability for a therapist to certify or know whether the client is adhering to treatment, an LMS was an ideal tool to empower therapists to better understand client commitment to treatment and also understand a client's specific issues as documented in the LMS.

Although some institutions and developers may extrapolate from our work with TAO that the therapeutic process is ripe for complete automation, we would caution against this approach. Research has indicated that if the therapist-client relationship is at all similar to the teacher-student relationship, generic scaffolds, such as TAO, in educational settings would likely be ineffective without a therapist as part of the implementation (McNeill \& Krajcik, 2009; Saye \& Brush, 2002). At this time, our team hypothesizes that a client's belief in the watchful eye of the therapist is having a positive impact upon treatment adherence and contributes to the therapeutic alliance. The impact and importance of the live videoconference with a therapist cannot be underestimated. Without a therapist, we would expect to have outcomes similar to other self-help materials or the analogous massive open online courses (MOOCs), where learner attrition is high (Breslow et al., 2013; Liyanagunawardena, Adams, \& Williams, 2013).

\section{Cognitive Task Analysis}

The design of therapeutic scaffolds requires an intensive cognitive task analysis of the types of processes and question paths that therapists commonly employ to improve client outcomes. Cognitive task analysis is a means to develop expert systems and to understand critical decision points and strategies used by experts to solve complex problems, such as the therapeutic process (Clark, Feldon, van Merriënboer, Yates, \& Early, 2008). In the case of TAO, the instructional designer was able to interview and work closely with the subject matter expert (SME) who provided paths or treatment scenarios that could be used to develop therapeutic scaffolds. To a great degree, the instructional designer relied upon the SME's extensive experience with the client population to create heuristics that could be coupled with the instructional scaffolds. The use of heuristics is well documented within medical applications and widely understood to be a pragmatic approach to solving complex problems for commonly occurring problems (Gigerenzer, 2004).

Students as Content Critics 
One portion of the development process consisted of inviting students to view and comment on the video content and user interface development, both individually and as a group. While the content was presented in an online classroom format, the goal was broader: Students should not only learn the principles and techniques to understand and manage their anxiety but also be encouraged to practice exercises that would help them identify and reduce their anxiety. The goal of encouraging students to practice what they learned meant that relatability was an important factor; thus, students whose ages and situations would be similar to those using the anxiety treatment were asked to review the content.

In this particular development process, an undergraduate peer educator group for the university counseling center was solicited for feedback. The group consisted of 20 undergraduate ambassadors whose purpose was to educate other students at the university regarding mental health issues and to reduce stigma surrounding mental health. Students applied to and were selected for this program and attended a rigorous training program throughout the year designed to help them educate other students in an engaging manner while also learning about mental health issues and services of the university. In addition to their role of serving as ambassadors and educators to the university community, the group has also, on numerous occasions, acted in an advisory role to the counseling center in development of presentation of services and new programming. The group's knowledge of mental health issues, as well as their direct experience as students who understand the values and interests of their age group, made them well suited for providing feedback during this development process. In addition, it also served as early training for them so that they would be able to promote the treatment to groups on campus.

The group was asked to review portions of content during one of their regular weekly meetings. While an already-formed group may have disadvantages in terms of variety of members, it can provide convenience when limited time or resources are available for development. In the group review, members were asked to watch educational video sessions that had been filmed and edited by a third party and to provide feedback while they were watching and also after the viewing. Reviewers were asked for specific feedback in their overall impression of the video content: how they felt about the content; how relatable the content was to the average student's life; and what changes they would make to wording and/or presentation of the material. Following the presentation of the video material, students were encouraged to discuss their impressions with each other and with the developer, who took notes during the process and provided this information to the rest of the development team during a later development team meeting. In addition to reviewing the video content, the peer educators were also asked their opinions on a reworking of the user interface, particularly the presentation scheme.

Finally, the peer educator group served as a resource to solicit individual subjects for a usability study. In this instance, a volunteer from the peer educator group was solicited and engaged in using the online materials while being observed by a member of the development team, who watched for any issues that may have arisen, such as confusion in navigating through the menu system or entering any data into the system.

Overall, the convenience of the already-existing peer educator group helped move development along at a reasonable pace, rather than slowing development down while a group was formed and volunteers were found. In addition, the use of students provided valuable feedback in ensuring that the material was relatable for students using the system. While this type of feedback loop may not be permissible in the traditional university e-learning environment, it was essential in this case since our goal was to both educate clients in CBT practices and also not make TAO appear to be a traditional course, which might trigger anxiety and stress.

\section{Reflection on Outcomes}

Benton, Heesacker, Snowden, and Lee (in press) examined the effectiveness of TAO in reducing anxiety symptoms and enhancing the psychological functioning of college students, as compared to those receiving individual face-to-face counseling. Results revealed that TAO clients experienced greater improvements in global mental health, well-being and life functioning, and anxiety symptom reduction than students receiving face-to-face individual counseling (Benton et al., in press). The authors concluded that these results were comparable to recent meta-analyses and randomized controlled trials supporting 
the efficacy of therapist-assisted interventions for anxiety and depression (e.g., Andersson \& Cuijpers, 2009; Andrews et al., 2010; Klein et al., 2006). Although the empirical factors impacting mental health outcomes and client satisfaction remain to be clarified through systematic study, the TAO program does appear to be working for both the clients and therapists who have volunteered to participate in the program.

For practitioners interested in replicating or modifying the approach taken in the TAO program, it is advisable to collaborate with an instructional design team and to identify clinical faculty interested in a team-based and collaborative project. One special consideration is the need to customize an LMS to meet privacy needs and to restrict all access to client data systematically. We recommend placing the server on a private network that can only be accessed through a virtual private network (VPN) and the systematic training of all individuals in HIPAA compliance. In addition, the LMS will need to be "cleaned" of all references to grades, quizzes, and any other traditional references to an academic course since many of these student clients suffer anxiety specifically with respect to academic activities. This is easy to achieve through customization of the LMS language pack and other edits to the LMS's CSS/HTML. In our particular case, we used Moodle since it was open source and the development team was familiar with its potential. We used Articulate Storyline to produce the psychoeducational materials as SCORM packages, and we deployed these SCORM packages through Moodle's SCORM 1.2 player and then moved to Rustici software's Engine product to take advantage of the more robust data collection capabilities of SCORM 2004.

What separates the TAO approach from mere video instruction or self-help is a clear awareness of the need to create both interactive scaffolds that engage awareness and also systems that allow therapists to supervise actual client adherence to treatment. Practitioners engaged in the creation of therapeutic psychoeducational materials are advised to build clinical prompts that walk the client through the therapy process, allow for reflection that is guided by a clear clinical framework, and provide clients with tools to practice monitoring, relaxation, and exposure techniques in real time.

While the design and delivery of traditional educational content will continue to be the primary focus for e-learning units, TAO represents an opportunity for university or college instructional units to engage in cross-unit collaboration with campus wellness and student support services. High-quality instructional design in the area of student support services can become an integral part of every institution's mission to better serve the needs of the student body. Some postsecondary schools will have the resources to replicate and improve upon this model in cases where IT infrastructure, instructional design units, and college counseling centers can come together to create HIPAA-compliant interventions to serve the student population. For schools without access to these resources, there are few options that directly apply to the needs of the college counseling center. One commercial product designed for college counseling centers is offered through TAOConnect at taoconnect.org that has licensed the platform and psychoeducational materials from the University of Florida that were used at the Counseling and Wellness Center.

Telemental health is an exciting area for college counseling centers and is expected to grow in the years to come. Future applications will include new treatments for depression and attention disorder, along with interesting blended approaches to therapy. The Counseling and Wellness Center at the University of Florida hopes to continue our exploration and experimentation with technological solutions that may lead to more effective student support. 


\section{References}

Allen, M., \& Sites, R. (2012). Leaving ADDIE for SAM: An agile model for developing the best learning experiences. American Society for Training and Development.

American College Health Association. (2014, Spring). National college health assessment: Undergraduate reference group executive summary. Retrieved from http://www.acha-ncha.org/docs/ACHANCHA-II_ReferenceGroup_ExecutiveSummary_Spring2014.pdf

Andrews, G., Cuijpers, P., Craske, M. G., McEvoy, P., \& Titov, N. (2010). Computer therapy for the anxiety and depressive disorders is effective, acceptable and practical health care: A metaanalysis. PLOS ONE, 5(10): e13196. doi:10.1371/journal.pone.0013196

Andersson, G., \& Cuijpers, P. (2009). Internet-based and other computerized psychological treatments for adult depression: A meta-analysis. Cognitive Behavioral Therapy, 4, 196-205.

Belland, B. R. (2014). Scaffolding: Definition, current debates, and future directions. In J. M. Spector, M. D. Merrill, J. Elen, \& M. J. Bishop (Eds.), Handbook of research on educational communications and technology (pp. 505-518) New York: Springer.

Benton, S., Heesacker, M., Snowden, S., \& Lee, G. (in press). High-engagement, therapist-assisted, online (TAO) psychotherapy for anxiety in college.

Berger, T., Caspar, F., Richardson, R., Kneubuehler, B., Sutter, D., \& Andersson, G. (2011). Internetbased treatment of social phobia: A randomized controlled trial comparing unguided with two types of guided self-help. Behaviour Research Therapy, 49, 158-169.

Breslow, L., Pritchard, D. E., DeBoer, J., Stump, G. S., Ho, A. D., \& Seaton, D. T. (2013). Studying learning in the worldwide classroom: Research into edX’s first MOOC. Research \& Practice in Assessment, 8(1), 13-25.

Butler, A. C., Chapman, J. E., Forman, E. M., \& Beck, A. T. (2006). The empirical status of cognitivebehavioral therapy: A review of meta-analyses. Clinical Psychology Review, 26, 17-31.

Clark, R. E., Feldon, D., van Merriënboer, J. J., Yates, K., \& Early, S. (2008). Cognitive task analysis. Handbook of Research on Educational Communications and Technology, 3, 577-593.

Walter, D., Carey, L., \& Carey, J. (2014). The systematic design of instruction (8th ed.). Upper Saddle River, NJ: Pearson Education, Inc.

Gallagher, R. P. (2011). National survey of counseling center directors (Monograph Series No. 8T). Alexandria, VA: The International Association of Counseling Services, Inc. Retrieved from http://collegecounseling.org/wp-content/uploads/2011-NSCCD.pdf

Gigerenzer, G. (2004). Fast and frugal heuristics: The tools of bounded rationality. In D. J. Koehler \& N. Harvey (Eds.), Blackwell Handbook of Judgment and Decision Making, (pp. 62-88). Malden, MA: Blackwell Publishing.

Jonassen, D. H. (1999). Designing constructivist learning environments. In C. Reigeluth (Ed.), Instructional Design Theories and Models: A New Paradigm of Instructional Theory (1st ed., Vol. 2, pp. 215-239). New York: Routledge. 
Liyanagunawardena, T. R., Adams, A. A., \& Williams, S. A. (2013). MOOCs: A systematic study of the published literature 2008-2012. The International Review of Research in Open and Distributed Learning, 14(3), 202-227.

Mayer, R. E. (2009). Multimedia learning (2nd ed.). New York: Cambridge University Press.

McNeill, K. L., \& Krajcik, J. (2009). Synergy between teacher practices and curricular scaffolds to support students in using domain-specific and domain-general knowledge in writing arguments to explain phenomena. The Journal of the Learning Sciences, 18(3), 416-460.

Morrison, G. R., Ross, S. M., Kemp, J. E., \& Kalman, H. (2012). Designing effective instruction (7th ed.). John Wiley \& Sons.

Mureşan, V., Montgomery, G. H., \& David, D. (2012). Emotional outcomes and mechanisms of change in online cognitive-behavioral interventions: A quantitative meta-analysis of clinical controlled studies. Journal of Technology in Human Services, 30(1), 1-13.

Puntambekar, S., \& Hubscher, R. (2005). Tools for scaffolding students in a complex learning environment: What have we gained and what have we missed? Educational Psychologist, 40(1), $1-12$.

Reetz, D. R., Barr, V., \& Krylowicz, B. (2013). The Association of University Counseling Center Directors annual survey. Retrieved from http://files.cmcglobal.com

AUCCCD_Monograph_Public_2013.pdf

Saye, J. W., \& Brush, T. (2002). Scaffolding critical reasoning about history and social issues in multimedia-supported learning environments. Educational Technology Research and Development, 50(3), 77-96.

Spek, V., Cuijpers, P., Nyklicek, I., Riper, H., Keyzer, J., \& Pop, V. (2007). Internet-based cognitive behaviour therapy for symptoms of depression and anxiety: A meta-analysis. Psychological Medicine, 37, 319-328.

Travers, M. F., \& Benton, S. (2014). The acceptability of therapist-assisted, internet-delivered treatment for college students. Journal of College Student Psychotherapy, 28(1), 35-46. 